\title{
Reliability of a new rapid step test for older women and its relationship with fall risk and leg muscle function
}

\author{
Shunsuke Yamaji ${ }^{1^{*}}$, Shinichi Demura ${ }^{2}$, Sohee Shin $^{3}$, Masanobu Uchiyama $^{4}$ \\ ${ }^{1}$ Faculty of Medical Sciences, University of Fukui, Fukui, Japan; \\ *Corresponding Author: yamaji@u- fukui.ac.jp \\ ${ }^{2}$ Graduate School of Natural Science and Technology, Kanazawa University, Kanazawa, Japan \\ ${ }^{3}$ School of Medicine, Gifu University, Gifu, Japan \\ ${ }^{4}$ Akita Prefectural University, Akita, Japan
}

Received 1 August 2012; revised 1 September 2012; accepted 9 September 2012

\begin{abstract}
This study aimed to examine trial-to-trial reliability of a new clinical test, rapid reaction step cued on a screen test, and its relationship with fall risk score, independence in activity of daily living score, and various leg muscle function tests. One-hundred fifty seven older women participated in this study. Nine square plates $(32 \times$ $32 \mathrm{~cm}$ ) were lined up with three plates. Participants stood on a center plate and were instructed to step, using one leg, to one of the other eight plates, as quickly as possible, when that plate changed in color from white to red. The mean total time and the mean total error time of rapid reaction step cued on a screen and, fall risk score, activity of daily living score, lower muscle function (isometric muscle strength: toe flexion, plantar flexion, knee extension, hip flexion; balance: one leg standing time with eye open, functional reach test; gait: $\mathbf{1 0} \mathrm{m}$ walk time with maximal speed). Results: trial-to-trial reliabilities of step parameters were high (intraclass correlation coefficient $[I C C]=0.75-0.85$ ). The step parameters correlated significantly with the other parameters except for toe flexion and hip flexion strengths. In conclusion, the present step test was found to evaluate physical function related to prevention of falls in older people.
\end{abstract}

Keywords: Fall Risk; Older Person; Balance; Mobility

\section{INTRODUCTION}

In most countries with an aging society, the primaryprevention of bedridden and other elderly persons unable to care for themselves was critical issue. To achieve successful aging, emphasis should be placed on improving independence in daily living rather than seeking perfect health. Falls in older people produce physical and psychological trauma, loss of independence, or even death, and is a serious problem in terms of decreasing quality of life [1].

Falls occur due to a complex combination of factors such as physiological function level, visual acuity and hearing, disease and disorder, cognitive function, medication, home environment, and age $[2,3]$. On the other hand, fall prevention intervention for the older living in self-reliant attach importance to improve physical fitness related fall prevention.

Physical function is a modifiable risk factor in every older individual and contributes greatly to falls. Particularly, it was reported that a decrease in leg muscle strength, gait and balance ability all have a higher relative risk ratio ( $R R=4.4,2.9$, and 2.9 , respectively) [2]. In addition, many tests that evaluate these physical function levels have been proposed. Mobility was evaluated by the $10 \mathrm{~m}$ walk test [4], Tandem gait test [5] which walks in the toes of the back foot touch the heel of the front foot at each step, figure eight track walk test [6], Timed up \& Go test [7,8], Obstacle walk test [9], and Maximal stepping test [10-12]. Balance ability was evaluated by functional reach [13], and one-leg standing with open eye [14]. Leg muscle strength was evaluated by isometric strength and the sit-to-stand test $[15,16]$. These test performances are not only useful for evaluating the independence in daily-living activities of older persons, but can also be used as a training tool for improving physical function.

However, falls occur when a stable body posture cannot be maintained due to accidental disturbance [1]. It is very difficult to simulate fall-inducing accidental distur- 
bances in these tests because they are voluntarily performed as a single task based on prospective movement $[12,17]$. An effective strategy to avoid falls is a stepping strategy, which extends a base of support when body sway cannot be controlled by ankle and hip strategies [18]. Maki et al. [18] examined stepping ability by physical disturbance using floor tilt, floor vibration, and traction apparatus, but they had problems with respect to practicability and safety using these techniques as a general exercise or an evaluation test.

The stepping strategy of avoiding a fall requires rapid postural control and quick step. From this view point, previous studies proposed several voluntary stepping tests, such as repeated stepping on the spot with maximal effort [10-12] and stepping to forward, backward, left and right directions (Four square stepping) $[19,20]$. However, they are also insufficient for assessing the stepping strategy since they are limited to a single task. Melzer et al. [21] proposed a voluntary step execution test under dual task, performing a modified-Stroop test. Shin and Demura [22] and Demura et al. [23] proposed new step tests with a relatively safe task in which older people step with slower fixed tempos than a usual walking tempo. A step adjusted to a fixed tempo, with extension of one leg during the support phase, demands maintaining body balance and correct tempo step. The use of dual or secondary tasks (Stroop test or keeping pace with the tempo) in addition to the main movement (stepping), as used in the above tests, have been used to evaluate the attention required in balance control. The premise is that the individuals performing two tasks simultaneously require more than the total information processing capacity, and performances of either or both tasks generally decrease.

However, the tempo step test is not enough of a disturbance stimulus for assessing stepping strategy, because the step patterns are determined in advance. In addition, avoiding a fall depends not only on a person's sensory motor function and balance ability, but also on a perception of dangerous signs of falling and the proper responses to keep body posture [24]. The perception required for body control does not demand complex cognitive processes as in a Stroop test. Rather, the dual task test to assess fall avoidance is thought to involve a simple selective reaction.

Based the above, we developed a new step test which requires participants to rapid step in random directions along with a fixed tempo (rapid reaction step cueing by a screen), as a balance task combined with a visuospatial secondary task would properly evaluate the ability to avoid falls.

It is hypothesized that the reliability of the step test are good, and can evaluate both the fall risk scores and independence in activity of daily living scores, rather than various other leg muscle functions. Thus, the step test may be useful to evaluate the ability of fall avoidance in a clinical setting.

This study aimed to examine the trial-to-trial reliability of this test and its relationship with the fall risk score, independence in activity of daily living score, and various leg muscle function tests in older women.

\section{METHODS}

\subsection{Participants}

One-hundred fifty seven older women aged $65-87$ years (Age: $72.0 \pm 6.7$ years, Height: $148.3 \pm 6.3 \mathrm{~cm}$, Body mass: $51.9 \pm 6.3 \mathrm{~kg}$ ) living independently in a community dwelling participated in this study. The participants were selected based on the following criteria: ambulatory without walking aid and no visual problem in visible colors. The participants have taken part in a weekly light exercise program (stretch exercise for 15 minutes) for over a year. Informed consent was obtained from each participant after a full explanation of the experimental project and its procedure. This study received approval from the Kanazawa University ethical committee.

\subsection{Rapid Reaction Step Cued on a Screen Test}

Figure 1 shows the rapid reaction step cued on a screen test (step test). The device consisted of nine square plates $(32 \times 32 \mathrm{~cm})$, with three plates placed in every direction at $5 \mathrm{~cm}$ intervals. Participants were instructed to step onto a plate as quickly as possible when the corresponding squares on the screen changed from white to red according to a step pattern at $40 \mathrm{bpm}$ tempo. The device measured the reaction time using a sampling frequency of $100 \mathrm{~Hz}$ of the subject stepping a correct plate in response to cueing by the screen, and time required to step (time from leaving the plate to contacting on another plate). A seventeen-inch screen was set at $2 \mathrm{~m}$ distance in front of participants with cells directly representing the plates making contact on the ground.

Figure 2 shows the step pattern used in this study. Participants stood on the center plate (home position), and stepped using one leg to one of eight plates as indicated on the screen, and then returned to the home position. The step protocol consisted of twenty steps presented at 40 bpm intervals. The trial time was 30 seconds. The participants' performance might have been affected by the memorization of the sequence because the steps to the home position were performed on every second step. However, this study selected the above step protocol to use the same number of steps by the right and the left legs.

Considering the effect of memorization, the mean of the total time required among every step (mean time required among every step) and the mean of the total error 


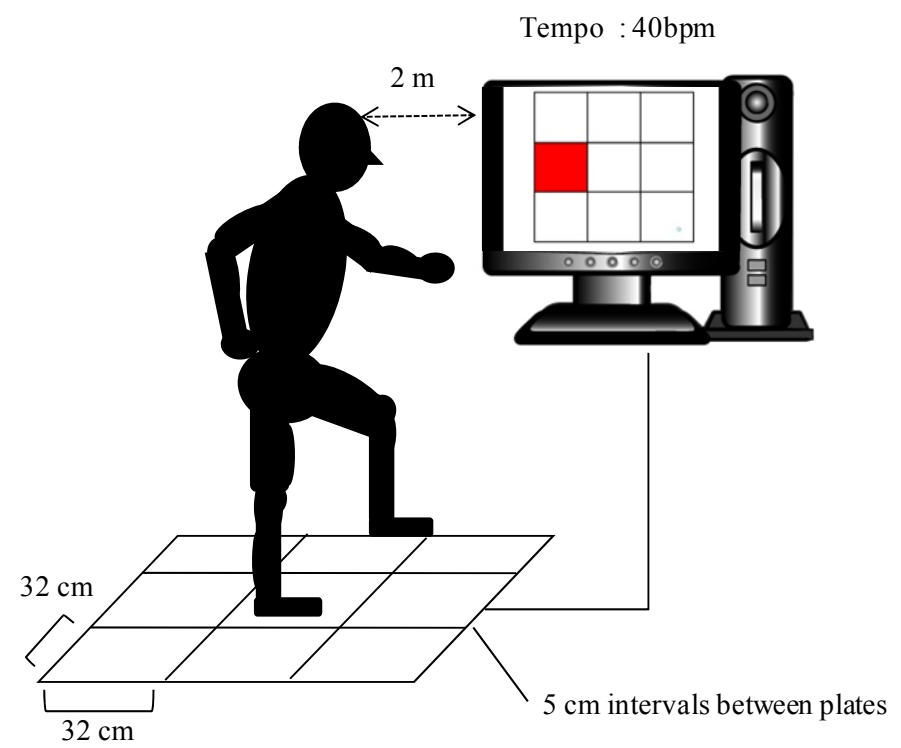

Figure 1. Rapid reaction step cueing by the screen test.

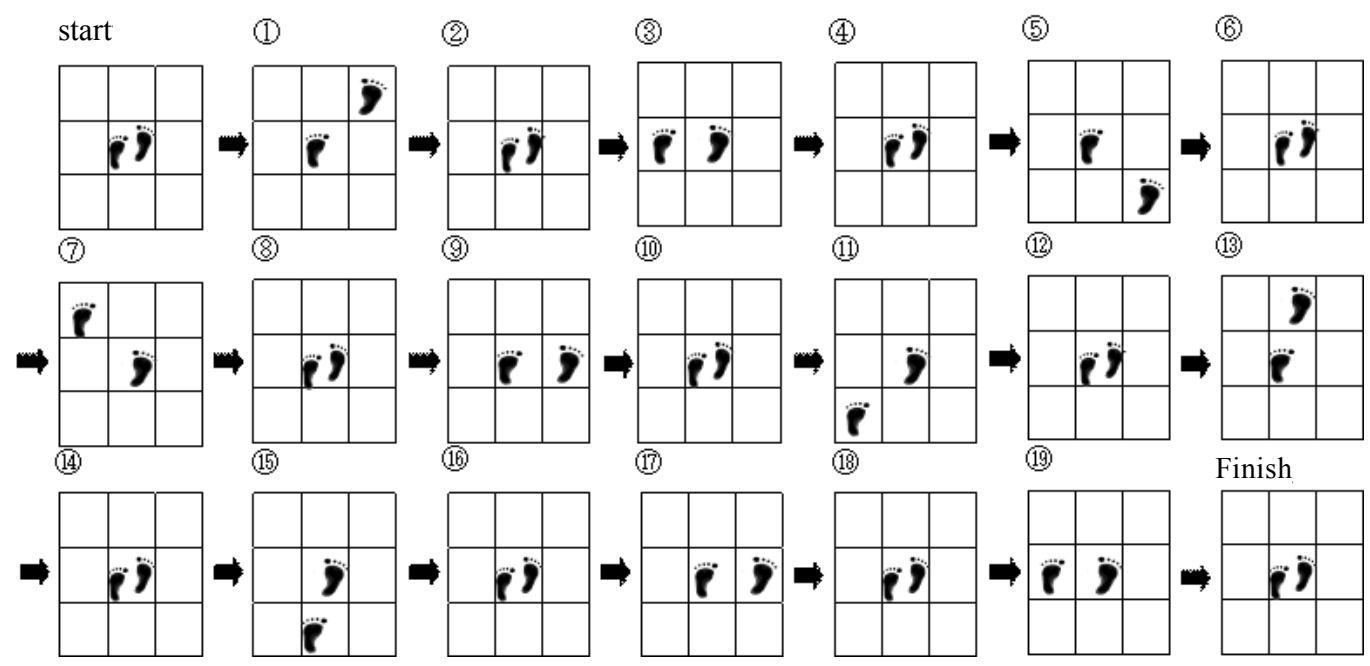

Figure 2. Step pattern. The center plate (start position) was defined as home position.

time between foot contacts and cueing time (mean error time) were selected as step parameters. Moreover, both parameters were calculated according to the step from home position (10 steps) and to home position (10 steps).

After the participants practiced a step test two or three times to acquaint themselves with the exercise, they carried out two trials with three minute rest intervals. They were instructed to step on a certain indicated plate and to re-step to the correct plate if stepping on a wrong plate.

\subsection{Falls Risk Assessment Score and Independence of Daily Living Score}

Fall risk was estimated using a fall risk assessment questionnaire [25]. This consisted of eight risk factors (gait deficit, balance deficit, muscle weakness, disease, medication use, environment, visual and hearing deficit, and fall fears: 15 items). Participants answered all questions with a dichotomous scale (yes or no). The response with a high risk category for each question was considered to be a "high-risk response", and persons with a score over five points were judged to have a high fall risk. The result of discriminant analysis using fall experience as a dependent variable and the total score of this questionnaire showed that the percentage of distinguishing correctly between fallers and non-faller was $84.7 \%$ [26].

Independence in daily living was estimated in terms of physical function level by a fall efficacy scale [27] and the activity of daily living (ADL) test [28]. The former consisted of 13 items regarding activities of daily living, and participants evaluated their confidence with a scale of one to ten. The test-retest reliability was very high 
$(\mathrm{ICC}=0.97)[27]$.

The ADL test consists of 12 items representing ADL domains of walking ability, changing and holding posture, balance, muscular strength, and dexterity (manual activity). Test-retest reliability of the ADL index showed significant and high correlations in 12 ADL items $(r=0.67$ $0.89)$ and the overall score $(r=0.94)$. The ADL score was significantly related to age and the physical fitness test scores, and tended to be higher in subjects with a higher self-assessment of health status and physical fitness level, and who exercised more [29]. Table 1 shows the mean scores of each questionnaire in this study.

\subsection{Leg Muscle Function Tests}

Leg muscle function tests were selected tests of leg muscle strength, balance ability, and gait, which were related to mobility and body posture control ability (isometric muscle strength: toe flexion, plantar flexion, knee extension, hip flexion; balance: one leg standing time with eye open, functional reach test; gait: $10 \mathrm{~m}$ walk time with maximal speed). Isometric muscle strength tests were measured twice in both legs by a hand-held dynamometer ( $\mu$ TAS F-1, Anima, Japan). The mean maximal strength of each joint in both legs was used. Plantar flexion was measured in a long sitting position, and the others were measured in a sitting on a chair. Functional reach was measured by using an elastic stick [30]. Each participant maximally extended the dominant hand from an upright posture while touching the top of an elastic stick fixed at the dominant acromion height on the wall. They pushed and shortened the elastic stick with the extended dominant hand. The shortened distance of the elastic stick was then measured.

\subsection{Data Analysis}

Trial-to-trial reliability of step parameters was examined by intra-class correlation coefficients (ICC). Intraclass correlation coefficients less than 0.5 represent poor reliability; 0.5 to 0.75 indicates fair reliability; and over 0.75 demonstrates good reliability $[31,32]$. One-way repeated measures ANOVA were used to reveal the mean differences of step parameters between trials. Pearson's correlation coefficient was calculated to reveal relationships among step parameters, fall risk assessment score, independent of daily living score, and leg muscle function. Pearson's correlation (r) less than 0.4 represent poor correlation; 0.4 to 0.7 indicates moderate correlation; and over 0.7 demonstrates high correlation [33]. A probability level of 0.05 was indicative of statistical significance.

\section{RESULTS}

Table 2 shows intra-class correlation coefficients (ICC) of step parameters. There were significant differences between trials except for mean error time of the step from the home position. The mean time required among every step for the step from home position was significantly longer, and that for the step to home position was significantly shorter. ICCs were over 0.75 (ICC $=0.75$ 0.85 ). A mean of two trials was used for further analysis.

Table 3 shows the correlations among step parameters, fall risk assessment score, fall efficacy scale, activity of daily living score, and leg muscle function. The correlations between mean error times during the total step and the step from home position, and between mean times required during the step from the home position and the step to home position were significantly high $(\mathrm{r}=0.94$ and 0.95 , respectively). The step parameters correlated significantly with the other parameters except for toe flexion and hip flexion strengths $(|\mathrm{r}|=0.20-0.54, \mathrm{p}<$ 0.05 ). The leg muscle function correlated significantly with fall risk assessment score (except for knee extension strength), fall efficacy scale, and activity of daily living score, but they tended to be slightly lower compared with correlations between the mean error time for those and the total step.

\section{DISCUSSION}

Leg muscle strength, balance, and walking ability contribute greatly to fall prevention and independence in daily living in older people $[34,35]$. Hence, their physical function retraining has focused almost exclusively on their function improvement. However, the major factors related to falls are losing balance due to false stepping or slipping during ambulation. Hence, postural control during an unstable supporting phase and quick response

Table 1. Mean score in each questionnaire.

\begin{tabular}{ccc}
\hline & Mean & SD \\
\hline Falls risk assessment score & 3.0 & 1.7 \\
Fall efficacy scale & 99.5 & 33.8 \\
Activity daily living score & 26.8 & 4.9 \\
\hline
\end{tabular}

Table 2. Trial-to-trial reliability of step parameters.

\begin{tabular}{cccccccccc}
\hline & \multicolumn{1}{c}{ 1st trial } & \multicolumn{2}{c}{ 2nd trial } \\
\cline { 3 - 8 } & & Mean & SD & Mean & SD & F $(1,156)$ & P & ICC \\
\hline $\begin{array}{c}\text { Mean error time } \\
\text { Total step }\end{array}$ & (s) & 0.72 & 0.32 & 0.64 & 0.34 & 14.51 & 0.00 & 0.85 \\
$\begin{array}{c}\text { The step from } \\
\text { home position }\end{array}$ & (s) & 1.01 & 0.27 & 0.97 & 0.29 & 2.89 & 0.09 & 0.75 \\
$\begin{array}{c}\text { Mean time required } \\
\text { among every step } \\
\begin{array}{c}\text { The step from } \\
\text { home position }\end{array}\end{array}$ & (s) & 1.96 & 0.26 & 2.02 & 0.19 & 20.64 & 0.00 & 0.83 \\
$\begin{array}{c}\text { The step to home } \\
\text { position }\end{array}$ & (s) & 0.89 & 0.24 & 0.83 & 0.20 & 21.64 & 0.00 & 0.85 \\
\hline
\end{tabular}




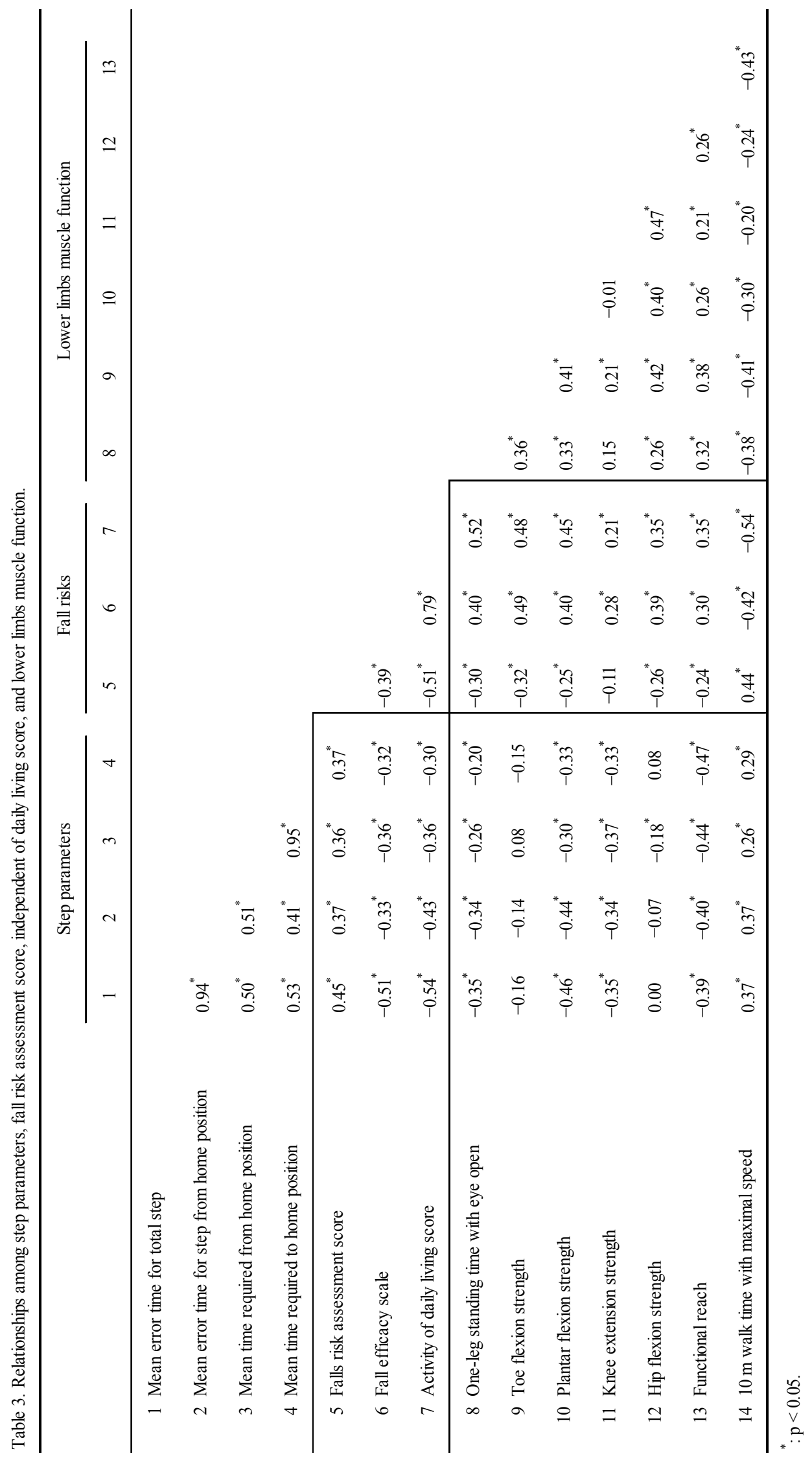


ability are very important for fall prevention, but the retraining and assessment methods to improve them have been little examined. We proposed new physical function retraining and assessment methods for fall prevention in older people who respond and step rapidly to the screen and control step tempo.

All the present participants with relatively high independence could perform the step test at a tempo of 40 $\mathrm{bpm}$ without having to reduce the tempo. However, 11 out of 157 participants retried the test because of losing balance during a trial. In the preliminary experiment using $60 \mathrm{bpm}, 6$ out of 10 participants could not keep up with the tempo. Shin and Demura [22,36,37] reported that a time difference in the step test on the spot with stipulated tempo of $40 \mathrm{bpm}$ showed greater individual differences than that with 60 and $120 \mathrm{bpm}$, and showed a difference between the different fall-risk groups. Hence, it is believed that older people who can walk independently can adequately perform the step test at a tempo of $40 \mathrm{bpm}$.

Reliability of step parameters was good (ICC: over 0.75). Demura et al. [23] reported that ICCs of the step test with maximal effort on the spot were over 0.9. Hashidate et al. [38] proposed the modified step test to measure the time required for repeated lateral stepping (5 times ) on $10 \mathrm{~cm}$ high plates, and reported that the ICCs were also over 0.9 in any direction. Moreover, Shin and Demura [22] reported that the ICC of the time difference between foot contact on the spot and a stipulated tempo of $40 \mathrm{bpm}$ was 0.73 . The reliability of the present step parameters was similar to or slightly lower than that in the above reports.

The step in this study has a high level of difficulty, compared to previous studies. Steps in previous studies permit the participant to select a stable step strategy because of motions prepared in advance; the steps in this study were performed under the dual task that required responding to external cueing, in addition to rapid and correct stepping. In short, this present step may increase the instability of step motion more than other transfer tests in which participants step or walk at their preferable or maximal speed. In this study, participants practiced two or three times in order to understand the motions before the step test.

In this study, participants practiced the task two or three times in order to understand the motions before the step test. However, trial differences-excluding mean error time for the step from the home position-were found, and the mean time required for the step to and the step from the home position differed significantly. From these results, the step strategy may not have been exactly stable between trials. Although the steps from home position are cued randomly, participants can predict the step to the home position because of it being designated as every second step. Although participants were not told that there is a certain step pattern of returning to the home position with every second step, the prediction effect by their memory cannot be discounted. Including predictable steps, the step strategy may have not been stable between trials. It will be necessary to examine which stepping strategy is stable under which trial numbers. It may also be necessary to examine the method of cueing all steps at random without setting a home position. Considering the above memory effect, the relationships between both mean error times and between both mean times required were examined by dividing them into the step from home position and the step to home position. The results were very positive $(r=0.94$ and 0.95 , respectively). Hence, step parameters may not be necessary to calculate parameters by dividing them into both step periods.

In this study, the leg muscles function except for knee extension strength showed significant relationships with fall risk assessment score, fall efficacy scale, and ADL score, but their relationships were somewhat lower than those between the mean error time of the total step. Many researchers have examined the relationships between falls in older people and the speed and stability of gait [39-41]. Kim et al. [42] reported that older people with a fall experience are more unstable in gait than those without a fall experience. Lee et al. [43] reported that a difference between groups that have or have not experienced a fall was found in the $2.45 \mathrm{~m}$ round-trip walk, $10 \mathrm{~m}$ gait time, cadence, and in one-leg standing time. Ikai et al. [44] reported that factors such as age, muscular strength, joints range of motion, motion control, and proprioceptive sensation influence gait, and that postural reflection and control (i.e., balance ability) are also closely involved in gait. In this study, the step parameters showed significant relationships with the gait time, functional reach, one-leg standing time with eyes open, and leg muscle strength except for toe flexion and hip flexion strengths. It is inferred that the present step parameters reflect comprehensively leg muscle function, and the instability of gait movement reflects the postural instability resulting from a longer one-legged support phase during the step test.

However, these correlations were not high. The step parameters do not strongly depend on the leg muscle functions, which reflect the voluntary muscle outputs, because the step in this study is performed under dual task (rapid and correct step, and selective reaction). That is, the step test engages with complex information processing as compared to the other leg function test. It is inferred that the capacity of rapid response to outside stimuli, as in this step, is important in avoiding falls. The assumption is exemplified by the result that fall risk and ADL scores correlated more highly with the step pa- 
rameters than with the other leg functions. Moreover, Yokoya et al. [45] reported that a low fall-risk group was superior to a high-risk group in one-leg standing time with eyes closed, but there were no significant differences in grip strength, repeated sit-up, sit and reach, 10 $\mathrm{m}$ walk time with obstacle, and sustained six-minute walk distance between both groups.

The fall-risk score may hardly reflect individual differences in physical function level, since many items (about $73 \%$ ) of factors (disease, medication use, environment, visual and hearing deficit, and fall fears) other than physical function are included in the fall-risk assessment questionnaire, and all questions are answered by a dichotomous scale (yes or no). However, the present step test would also be effective in determining fall risk in older people because of the significant relationship between them.

In this study, step movement was controlled only by stepping while quickly responding to cues on a screen, but not by the height of the elevated foot during a step and foot contact (e.g. foot contact with only toe part or whole sole). The control of step movement increases the difficulty of a test. For example, setting obstacles among the plates makes the one-leg support period during stepping longer due to requiring the foot to raise higher, and having the whole sole of the foot contact the plate makes the center of body gravity move greatly. This is meant to increase instability during stepping. It will also be necessary to examine this in a later study.

In conclusion, the step test in this study is useful to evaluate physical function related to fall prevention in older people. It may be used as an important indicator to determine their fall experience.

\section{REFERENCES}

[1] Oliver, D. (2007) Older people who fall: Why they matter and what you can do. British Journal of Community Nursing, 12, 500-507.

[2] Leipzig, R.M., Cumming, R.G. and Tinetti, M.E. (1999) Drugs and falls in older people: A systematic review and meta-analysis: II. Cardiac and analgesic drugs. Journal of the American Geriatrics Society, 47, 40-50.

[3] American Geriatrics Society, British Geriatrics Society, and American Academy of Orthopaedic Surgeons Panel on Falls (2001) Guideline for the prevention of falls in older persons. Prevention. Journal of the American Geriatrics Society, 49, 664-672. doi:10.1046/j.1532-5415.2001.49115.x

[4] van der Velde, N., Stricker, B.H., Pols, H.A. and van der Cammen, T.J. (2007) Withdrawal of fall-risk-increasing drugs in older persons: Effect on mobility test outcomes. Drugs and Aging, 24, 691-699. doi:10.2165/00002512-200724080-00006

[5] Gill, J., Allum, J.H., Carpenter, M.G., Held-Ziolkowska,
M., Adkin, A.L., Honegger, F. and Pierchala, K. (2001) Trunk sway measures of postural stability during clinical balance tests: Effects of age. The Journals of Gerontology, Series A: Biological Sciences and Medical Sciences, 56, 438-447. doi:10.1093/gerona/56.7.M438

[6] Graham, R.C., Smith, N.M. and White, C.M. (2005) The reliability and validity of the physiological cost index in healthy subjects while walking on 2 different tracks. Archives of Physical Medicine and Rehabilitation, 86, 20412046. doi:10.1016/j.apmr.2005.04.022

[7] Podsiadlo, D. and Richardson, S. (1991) The timed "Up \& Go": A test of basic functional mobility for frail elderly persons. Journal of the American Geriatrics Society, 39, 142-148.

[8] Newton, R.A. (1997) Balance screening of an inner city older adult population. Archives of Physical Medicine and Rehabilitation, 78, 587-591. doi:10.1016/S0003-9993(97)90423-8

[9] Rubenstein, L.Z., Josephson, K.R., Trueblood, P.R., Yeung, K., Harker, J.O. and Robbins, A.S. (1997) The reliability and validity of an obstacle course as a measure of gait and balance in older adults. Aging (Milano), 9, 127135.

[10] Medell, J.L. and Alexander, N.B. (2000) A clinical measure of maximal and rapid stepping in older women. The Journals of Gerontology, Series A: Biological Sciences and Medical Sciences, 55, 429-433. doi:10.1093/gerona/55.8.M429

[11] Cho, B.L., Scarpace, D. and Alexander, N.B. (2004) Tests of stepping as indicators of mobility, balance, and fall risk in balance-impaired older adults. Journal of the American Geriatrics Society, 52, 1168-1173. doi:10.1111/j.1532-5415.2004.52317.x

[12] Demura, S., Yamaji, S. and Kitabayashi, T. (2005) Gender and age-related differences of dynamic balancing ability based on various stepping motions in the healthy elderly. Journal of Human Ergology (Tokyo), 34, 1-11.

[13] Duncan, P.W., Weiner, D.K., Chandler, J. and Studenski, S. (1990) Functional reach: A new clinical measure of balance. Journal of Gerontology, 45, 192-197.

[14] Atwater, S.W., Crowe, T.K., Deitz, J.C. and Richardson, P.K. (1990) Interrater and test-retest reliability of two pediatric balance tests. Physical Therapy, 70, 79-87.

[15] Jones, C.J., Rikli, R.E. and Beam, W.C. (1999) A 30-s chair-stand test as a measure of lower body strength in community-residing older adults. Research Quarterly for Exercise and Sport, 70, 113-119.

[16] Yamada, T. and Demura, S. (2004) Influence of the relative difference in chair seat height according to different lower thigh length on floor reaction force and lower-limb strength during sit-to-stand movement. Journal of Physiological Anthropology, 23, 197-203. doi:10.2114/jpa.23.197

[17] Nnodim, J.O., Strasburg, D., Nabozny, M., Nyquist, L., Galecki, A., Chen, S. and Alexander, N.B. (2006) Dynamic balance and stepping versus tai chi training to improve balance and stepping in at-risk older adults. Journal of the American Geriatrics Society, 54, 1825-1831. 
doi:10.1111/j.1532-5415.2006.00971.x

[18] Maki, B.E., Perry, S.D., Scovil, C.Y., Peters, A.L., McKay, S.M., Lee, T.A., Corbeil, P., Fernie, G.R. and Mcllroy, W.E. (2008) Interventions to promote more effective balance-recovery reactions in industrial settings: New perspectives on footwear and handrails. Industrial Health, 46, 40-50. doi:10.2486/indhealth.46.40

[19] Dite, W. and Temple, V.A. (2002) A Clinical test of stepping and change of direction to identify multiple falling older adults. Archives of Physical Medicine and Rehabilitation, 83, 1566-1571. doi:10.1053/apmr.2002.35469

[20] Whitney, S.L., Marchetti, G.F., Morris, L.O. and Sparto, P.J. (2007) The reliability and validity of the four square step test for people with balance deficits secondary to a vestibular disorder. Archives of Physical Medicine and Rehabilitation, 88, 99-104. doi:10.1016/j.apmr.2006.10.027

[21] Melzer, I., Shtilman, I., Rosenblatt, N. and Oddsson, L.I. (2007) Reliability of voluntary step execution behavior under single and dual task conditions. Journal of Neuroengineering and Rehabilitation, 29, 4-16.

[22] Shin, S. and Demura, S. (2007) Effective tempo of the step test for dynamic balance ability in the elderly. Journal of Physiological Anthropology, 26, 563-567. doi:10.2114/jpa2.26.563

[23] Demura, S., Yamada, T. and Shin, S. (2008) Age and sex differences in various stepping movements of the elderly. Geriatrics \& Gerontology International, 8, 180-187. doi:10.1111/j.1447-0594.2008.00468.x

[24] St George, R.J., Fitzpatrick, R.C., Rogers, M.W. and Lord, S.R. (2007) Choice stepping response and transfer times: Effects of age, fall risk, and secondary tasks. The Journals of Gerontology, Series A: Biological Sciences and Medical Sciences, 62, 537-542. doi:10.1093/gerona/62.5.537

[25] Suzuki, T. (2001) Development and its use of a falling risk assessment chart for the elderly population. In: Health Assessment Committee, Ed., Health assessment manual, Kosei Kagaku Kenkyusho, Tokyo, 142-152.

[26] Demura, S., Sato, S., Yamaji, S., Kasuga, K. and Nagasawa, Y. (2011) Examination of validity of fall risk assessment items for screening high fall risk elderly among the healthy community-dwelling Japanese population. Archives of Gerontology and Geriatrics, 53, e41-e55. doi:10.1016/i.archger.2010.10.010

[27] Hellström, K. and Lindmark, B. (1999) Fear of falling in patients with stroke: A reliability study. Clinical Rehabilitation, 13, 509-517. doi:10.1191/026921599677784567

[28] Ministry of Education, Culture, Sports, Science and Technology of Japan (2000) Guideline for new physical fitness test.

http://www.mext.go.jp/a menu/sports $\% 20 /$ stamina/03040 901.\%20htm.\%20Accessed\%20March\%201,\%202012

[29] Demura, S., Sato, S., Kobayashi, H., Kasuga, K. and Toyoshima, Y. (1999) Development of ADL index for partially dependent older adults. Nihon Koshu Eisei Zasshi, 46, 25-34.
[30] Demura, S. and Yamada, T. (2007) Simple and easy assessment of falling risk in the elderly by functional reach test using elastic stick. The Tohoku Journal of Experimental Medicine, 213, 105-111. doi: $10.1620 /$ tjem.213.105

[31] Fleiss, J.L. (1981) Statistical method for rates and proportions. Wiley, Toronto.

[32] Portney, L.G. and Watkins, M.P. (2000) Foundations of clinical research: Applications to practice. 2nd Edition, Prentice Hall Health, Upper Saddle River.

[33] Sokal, R.R. and Rohlf, F.J. (2012) Biometry. 4th Edition, Freeman, New York.

[34] Creel, G.L., Light, K.E. and Thigpen, M.T. (2001) Concurrent and construct validity of scores on the Timed Movement Battery. Physical Therapy, 81, 789-798.

[35] Gregg, E.W., Cauley, J.A., Stone, K., Thompson, T.J., Bauer, D.C., Cummings, S.R. and Ensrud, K.E. (2003) Relationship of changes in physical activity and mortality among older women. JAMA: The Journal of the American Medical Association, 289, 2379-2386. doi:10.1001/jama.289.18.2379

[36] Shin, S. and Demura, S. (2009) The relationship of age and leg strength in the step test with stipulated tempo in the elderly. Archives of Gerontology and Geriatrics, 49, 311-316. doi:10.1016/j.archger.2008.11.009

[37] Shin, S. and Demura, S. (2009) Relationship between the step test with stipulated tempos and gait ability in the elderly. Journal of Physiological Anthropology, 28, 4954. doi:10.2114/jpa2.28.49

[38] Hashidate, H., Uchiyama, Y. and Shiomi, T. (2003) Development of modified step test as dynamic balance assessment: Reliability and validity. Rigakuryoho Kagaku, 19, 55-59. doi:10.1589/rika.19.55

[39] Smeesters, C., Hayes, W.C. and McMahon, T.A. (2001) Disturbance type and gait speed affect fall direction and impact location. Journal of Biomechanics, 34, 309-317. doi:10.1016/S0021-9290(00)00200-1

[40] van Iersel, M.B., Olde Rikkert, M.G. and Borm, G.F. (2007) A method to standardize gait and balance variables for gait velocity. Gait and Posture, 26, 226-230. doi:10.1016/j.gaitpost.2006.09.002

[41] Baczkowicz, D., Szczegielniak, J. and Proszkowiec, M. (2008) Relations between postural stability, gait and falls in elderly persons-preliminary report. Ortopedia, Traumatologia, Rehabilitacja, 10, 478-485.

[42] Kim, H., Yoshida, H., Suzuki, T., Ishizaki, T., Hosoi, T., Yamamoto, S. and Orimo, H. (2001) The relationship between fall-related activity restriction and fitness in elderly women. Nippon Ronen Igakkai Zasshi, 38, 805-811. doi:10.3143/geriatrics.38.805

[43] Lee, S., Kim, S., Park, Y. and Kim, J. (2007) Effects of falling experience to gait pattern, functional fitness and leg strength of elderly women. Korean Journal of Physical Education, 46, 369-378.

[44] Ikai, T., Tatsuno, H. and Miyano, S. (2006) Relationship between walking ability and balance function. Japanese Journal of Rehabilitation and Medicine, 43, 823-833. doi:10.2490/jijrm1963.43.828 
[45] Yokoya, T., Demura, S. and Sato, S. (2008) Fall risk characteristics of the elderly in an exercise class. Journal of Physiological Anthropology, 27, 25-32. doi:10.2114/jpa2.27.25 